\title{
$b$-hadron production in the general-mass variable-flavor-number scheme and the LHC data
}

\author{
G. Kramer $^{1}$ and H. Spiesberger ${ }^{2}$ \\ ${ }^{1}$ II. Institut für Theoretische Physik, Universität Hamburg, \\ Luruper Chaussee 149, D-22761 Hamburg, Germany \\ ${ }^{2}$ PRISMA Cluster of Excellence, Institut für Physik, Johannes Gutenberg-Universität, \\ 55099 Mainz, Germany, \\ and Centre for Theoretical and Mathematical Physics and Department of Physics, \\ University of Cape Town, Rondebosch 7700, South Africa
}

(Received 4 October 2018; published 13 December 2018)

\begin{abstract}
We study inclusive $b$-hadron production in $p p$ collisions at the LHC at different center-of-mass energies and compare with experimental data from the $\mathrm{LHCb}$ and CMS collaborations. Our predictions for cross sections differential in the transverse momentum and (pseudo)rapidity agree with data within uncertainties due to renormalization scale variations. A small tension is found if data and theory predictions are compared for cross section ratios at different center-of-mass energies.
\end{abstract}

DOI: 10.1103/PhysRevD.98.114010

\section{INTRODUCTION}

The investigation of inclusive production of hadrons containing $b$ quarks is particularly important to test quantum chromodynamics (QCD). The predictions in the framework of perturbative QCD are based on the factorization approach. Cross sections are calculated as a convolution of three basic parts: the parton distribution functions (PDFs) describing the parton content of the initial hadronic state, the partonic hard scattering cross sections computed as a perturbative series in powers of the strong coupling constant, and the fragmentation functions (FFs), which describe the production yield and the momentum distribution for a specified $b$ hadron in a parton. Since the $b$-quark mass is large and can often not be neglected compared with the transverse momentum, the cross section for $b$-hadron production depends on several large scales, which makes predictions very challenging.

In the past, measurements of inclusive $b$-hadron production and the corresponding QCD calculations were done first of all for the $B$ mesons, i.e., $B^{ \pm}, B^{0}, \bar{B}^{0}, B_{s}^{0}, \bar{B}_{s}^{0}$, but also $\Lambda_{b}^{0}$ and other $b$ baryons have been considered. Data for $p \bar{p}$ collisions at $\sqrt{S}=1.96 \mathrm{TeV}$ have been obtained at the FNAL Tevatron Collider [1,2] and for $p p$ collisions at $\sqrt{S}=5,7,8$ and $13 \mathrm{TeV}$ at the CERN Large Hadron Collider (LHC) by the ATLAS, CMS and LHCb

Published by the American Physical Society under the terms of the Creative Commons Attribution 4.0 International license. Further distribution of this work must maintain attribution to the author(s) and the published article's title, journal citation, and DOI. Funded by SCOAP ${ }^{3}$. collaborations [3-10]. First measurements of the production cross sections of $\Lambda_{b}^{0}$ baryons have been performed by the CMS Collaboration at the LHC [11] at $\sqrt{S}=7 \mathrm{TeV}$ and by the LHCb Collaboration for $\sqrt{S}=7$ and $8 \mathrm{TeV}$ [12].

Almost all of these data have been compared with nextto-leading-order (NLO) QCD predictions based on the so-called fixed-order next-to-leading-logarithm (FONLL) approach [13]. Data of the CMS, LHCb and ATLAS collaborations have also been compared with predictions obtained in the general-mass variable-flavor-number scheme (GM-VFNS) [14,15]. The GM-VFNS [16,17] (see also Ref. [18] for a more recent implementation of the GMVFNS) is similar to the FONLL scheme but contains different assumptions concerning fragmentation functions, and the transition to the fixed-flavor-number scheme (FFNS) in the low transverse momentum, $p_{T}$, region is treated in a different way. All comparisons between experimental data and theoretical predictions, whether based on the FONLL approach or on the GM-VFNS, show reasonable agreement within experimental uncertainties and taking into account the so-called theoretical error which is estimated by a variation of the factorization and renormalization scale parameters or the heavy quark masses.

An exception to agreement between experimental data and theoretical predictions had originally been reported by the LHCb Collaboration $[19,20]$. Their primary finding that the ratio $R_{13 / 7}$ of cross sections $d \sigma / d \eta$ as a function of the pseudorapidity $\eta$ in the region $2<\eta<5$ for $\sqrt{S}=13$ and $\sqrt{S}=7 \mathrm{TeV}$ did not agree with corresponding FONLL predictions [21] was corrected later [22] after a mistake in their detector simulation was found which changed the data for the $13 \mathrm{TeV} b$-quark production cross section. In the 
LHCb analysis, the observed semileptonic decays of $b$ hadrons was corrected in such a way that the extracted cross section can be interpreted as a measurement of the inclusive $b$-quark production, not just for one specific $B$-meson species.

Other measurements of $b$-quark production show some discrepancy between data and theoretical predictions, although with smaller significance than what was reported for the now corrected LHCb data. Among them we count the data from the CMS Collaboration for inclusive $B^{+}$production [10]. Here, $B^{+}$mesons were identified by their decay into $J / \psi K^{+}$final states at the energies $\sqrt{S}=7$ and $13 \mathrm{TeV}$. Differential cross sections were determined both as a function of $p_{T}$ (integrated over rapidity ranges $|y|<1.45$ and $|y|<2.1$ ), as well as a function of $|y|$ (integrated over $p_{T}$ in the ranges $10<p_{T}<100 \mathrm{GeV}$ and $17<p_{T}<$ $100 \mathrm{GeV})$. A discrepancy was observed in a comparison with predictions obtained in the FONLL approach.

The purpose of our present work is to present results for $b$-hadron production cross sections and the cross section ratios $R_{13 / 7}$ in $p p$ collisions at the LHC in the framework of the GM-VFNS. This framework is essentially the conventional NLO QCD parton-model approach supplemented with heavy-quark finite mass effects intended to improve the description at small and medium transverse momenta. First, we shall apply the GM-VFNS approach for the calculation of the NLO single-inclusive cross sections. Second, we shall investigate the dependence of the cross section ratio $R_{13 / 7}$ on the assumed input PDF. We will show that the cross section ratios have much smaller theoretical uncertainties than the differential cross sections themselves.

The outline of the paper is as follows. In the next section we introduce some details of the calculation, describe our choice of the proton PDFs and the fragmentation functions, and discuss how rapidity and pseudorapidity distributions are related. In Sec. III we collect our results for inclusive $b$-hadron cross sections $d \sigma / d \eta$ at 7 and $13 \mathrm{TeV}$ and compare with the LHCb data. A similar comparison with CMS data, including also the $p_{T}$ distribution, is performed in Sec. IV at 7 and $13 \mathrm{TeV}$ in the central rapidity region. In addition, we discuss theory predictions and experimental results for $J / \Psi$ production in Sec. V. Our conclusions with some outlook are presented in Sec. VI.

\section{SETUP, INPUT PDFs AND FFs}

The theoretical description of the GM-VFNS approach as well as the technical details of its implementation have been presented previously in Refs. [16,17]. Here we describe only the input required for the numerical evaluations discussed below.

As a default we use the proton PDF set CT14 [23] at NLO as implemented in the LHAPDF library [24]. To study the sensitivity on the PDF input we shall use two approaches. First we will use three alternative PDF sets: (i) HERA2.0 [25], (ii) MMHT [26] and (iii) NNPDF3.0 [27]. All of these PDF sets are NLO parametrizations; the last two of them are obtained from global fits to essentially the same experimental data as CT14, while the set HERA2.0 is based mainly on cross section data for deep inelastic scattering at HERA. Second, for the CT14 parametrization we will also study uncertainties obtained from variations of parameter eigenvalues. There are 56 members of the CT14 set corresponding to 28 pairs of eigenvalue variations. One pair is particularly interesting since it describes an enhanced/suppressed gluon distribution at very low $x$. The PDF uncertainty band is evaluated following the prescription given in Eq. (5) of Ref. [28] and corresponds to $90 \%$ C.L.

To describe the transition of $b$ quarks to $b$ hadrons we need nonperturbative FFs. We employ the $B$-meson FFs constructed in Ref. [29]. They are evolved at NLO and components for the transition from gluons and light quarks (including charm) to a $B$ meson are generated through Dokshitzer-Gribov-Lipatov-Altarelli-Parisi evolution. They were obtained by fitting experimental data for inclusive $b$ production in $e^{+} e^{-}$annihilation taken by the ALEPH [30] and OPAL [31] collaborations at CERN LEP1 and by the SLD Collaboration $[32,33]$ at SLAC SLC. These data were all taken on the $Z$-boson resonance. Therefore $\alpha_{s}^{\left(n_{f}\right)}\left(\mu_{R}\right)$ was evaluated with $n_{f}=5$ and the renormalization and factorization scales were fixed at $\mu_{R}=\mu_{F}=m_{Z}$. The starting scale was chosen to be $\mu_{0}=m_{b}=4.5 \mathrm{GeV}$. Below $\mu_{F}=\mu_{0}$ the light-quark and gluon FFs were assumed to vanish. A simple power ansatz gave the best fit to the experimental data.

One should notice that the $B$-meson FFs of Ref. [29] do not distinguish between different $b$-hadron final states. Both the OPAL [31] and the SLD [32,33] data include all $b$ hadrons, i.e., the mesons $B^{ \pm}, B^{0}, \bar{B}^{0}, B_{s}^{0}$ and $\bar{B}_{s}^{0}$ as well as $b$ baryons, while in the ALEPH analysis [30] only final states with identified $B^{ \pm}, B^{0}$ and $\bar{B}^{0}$ mesons were taken into account. Despite these differences in the experimental analyses it was assumed in Ref. [29] that all data can be described by one common FF. The resulting FF fit did indeed not show any significant difference with either of the two data sets, namely, OPAL and SLD including all $b$ hadrons on the one side, and ALEPH including only identified $B$ mesons on the other side. The FF was normalized to describe cross section data for $B^{+}$- and $B^{0}$-meson production. They can also be used to calculate the sum of all $b$-hadron states by removing the fragmentation fraction for the $b \rightarrow B^{ \pm}$transition, which was assumed as $f_{u}=f_{d}=0.397$ in Ref. [29].

For simplicity we shall take the initial- and final-state factorization scales entering the PDFs and FFs, respectively, to have the same value, denoted by $\mu_{F}$. The majority of data to which we are going to compare our theory predictions is dominated by low transverse momenta. For example, the LHCb data for the pseudorapidity distribution, $d \sigma / d \eta$, is integrated over all $p_{T}$, down to $p_{T}=0$. In a 
previous work [15] we have shown that the choice $\mu_{F}=\xi_{F} \sqrt{p_{T}^{2}+m_{b}^{2}}$ with $\xi_{F}=0.5$ for the factorization scale is appropriate in this case. This choice allows a transition to the fixed-flavor number scheme at finite values of the transverse momentum. The FFNS is the appropriate prescription for heavy-quark production in the range $p_{T} \lesssim m_{b}$. In the FFNS, subprocesses with heavy quarks in the initial state do not contribute. With our choice of $\mu_{F}$, the scale reaches the heavy-quark threshold $\mu_{F}=m_{b}$ already at $p_{T}=7.8 \mathrm{GeV}$ below which both the $b$ PDF and the FFs are zero. In Ref. [15] we have shown that with $\xi_{F}=0.5$ the cross sections $d \sigma / d p_{T}$ as a function of $p_{T}$ as measured by LHCb at $\sqrt{S}=7 \mathrm{TeV}$ [34] and by CDF at the Tevatron [1] can be described satisfactorily well down to $p_{T}=0$. The factor $\xi_{F}=0.5$ is not unique, but small variations like $\xi_{F}=0.4$ and 0.6 lead only to small changes, as shown in Ref. [15].

We evaluate the strong coupling $\alpha_{s}^{\left(n_{f}\right)}\left(\mu_{R}\right)$ as a function of the renormalization scale $\mu_{R}$ at NLO with $\Lambda_{\overline{\mathrm{MS}}}^{(4)}=$ $328 \mathrm{MeV}$ for $n_{f}=4$ flavors. This corresponds to $\Lambda \frac{(5)}{\mathrm{MS}}=$ $226 \mathrm{MeV}$ above the five-flavor threshold at $\mu_{R}=m_{b}$. For the $b$-quark pole mass we use $m_{b}=4.5 \mathrm{GeV}$ in accordance with the value chosen for the FF fit in Ref. [29]. This value is also compatible, though not identical, with the $b$-quark thresholds in the PDF parametrizations which we are going to use.

We have updated the implementation of our program for the calculation of GM-VFNS predictions to allow integration with fixed pseudorapidity. Results in our previous publications have always been given for rapidity intervals. We note that the difference between rapidity and pseudorapidity cross sections is quite substantial for the LHCb data. In Fig. 1 we show contours of constant pseudorapidity in the plane of transverse momentum $p_{T}$ and rapidity $y$. The calculation is made for the kinematic range relevant for the $\mathrm{LHCb}$ experiment to be discussed in the next section and using $m_{b}=4.5 \mathrm{GeV}$. One can see that at low $p_{T}$, where the dominating contribution to the cross section $d \sigma / d \eta$ is found, the average rapidity is shifted to much smaller values compared with the pseudorapidity. It turns out that $d \sigma / d \eta$ is increased, compared with $d \sigma / d y$, by $50 \%$ to $100 \%$ in the high- $\eta$ bins of the $\mathrm{LHCb}$ measurements while it is almost the same at smaller values of the (pseudo)rapidity. The effect on the cross section is not as strong as could be expected from Fig. 1 since the $\eta$ dependence of the differential cross section is not very strong, but the difference is still relevant. We also note that the results depend little on the exact choice of the particle mass in the relation between rapidity and pseudorapidity and our conclusions would not change if we had used a value corresponding to the lightest $b$ meson $\left(m_{B^{0}}=5.28 \mathrm{GeV}\right)$ or even the heavier $b$ hadron $\left(m_{\Lambda_{b}}=5.62 \mathrm{GeV}\right)$.

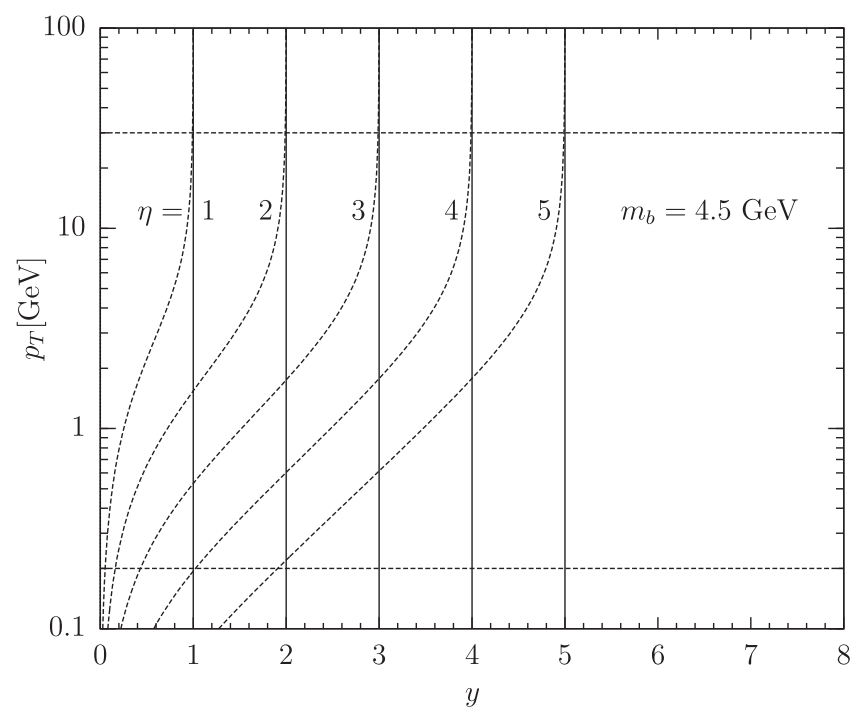

FIG. 1. Lines of constant pseudorapidity as a function of the rapidity for mass $m_{b}=4.5 \mathrm{GeV}$ in the $p_{T}$ range relevant for $\mathrm{LHCb}$ data. The horizontal dashed lines indicate the values of $p_{T}=0.2$ and $=30 \mathrm{GeV}$.

\section{COMPARISON WITH LHCb DATA}

The LHCb Collaboration has reported cross section measurements for $b$-hadron production in $p p$ collisions at $\sqrt{S}=7$ and $13 \mathrm{TeV}$ [22]. Results are given for the pseudorapidity dependence, $d \sigma / d \eta$, in six equal-sized bins in the range $2<\eta<5 . b$ hadrons are identified by their semileptonic decays into a ground-state charmed hadron in association with a muon. We show these data together with our results (full-line histograms) for the proton PDFs of CT14 [23] in Fig. 2. We have used the factorization scale $\mu_{F}=0.5 \sqrt{p_{T}^{2}+m_{b}^{2}}$ with $m_{b}=4.5 \mathrm{GeV}$ as in Ref. [15]. The renormalization scale was fixed as $\mu_{R}=\sqrt{p_{T}^{2}+m_{b}^{2}}$ and varied up and down by a factor of 2 to obtain an estimate of the theoretical uncertainty. This results in the dashed-line histograms in Fig. 2. The upper limit of the uncertainty band is found for $\mu_{R}=\mu_{F}=0.5 \sqrt{p_{T}^{2}+m_{b}^{2}}$ and the lower limit for $\mu_{R}=4 \mu_{F}=2.0 \sqrt{p_{T}^{2}+m_{b}^{2}}$. We do not observe a discrepancy between data and theoretical results: all data points lie inside the theoretical band for both values of $\sqrt{S}$ and in all $\eta$ bins. However, the shape of the $\eta$ dependence at values $\eta \lesssim 3$, where the cross section data decrease with decreasing $\eta$, is not visible in the theory prediction.

In Fig. 3 we show the ratio $R_{13 / 7}$ of the cross sections $d \sigma / d \eta$ for $\sqrt{S}=13$ and $7 \mathrm{TeV}$ and compare our results with the experimental values in the six $\eta$ bins. The figure shows predictions for the CT14 PDFs (full-line histogram). The theoretical result varies between 1.6 and 2.0. The uncertainty due to the variation of the renormalization scale 

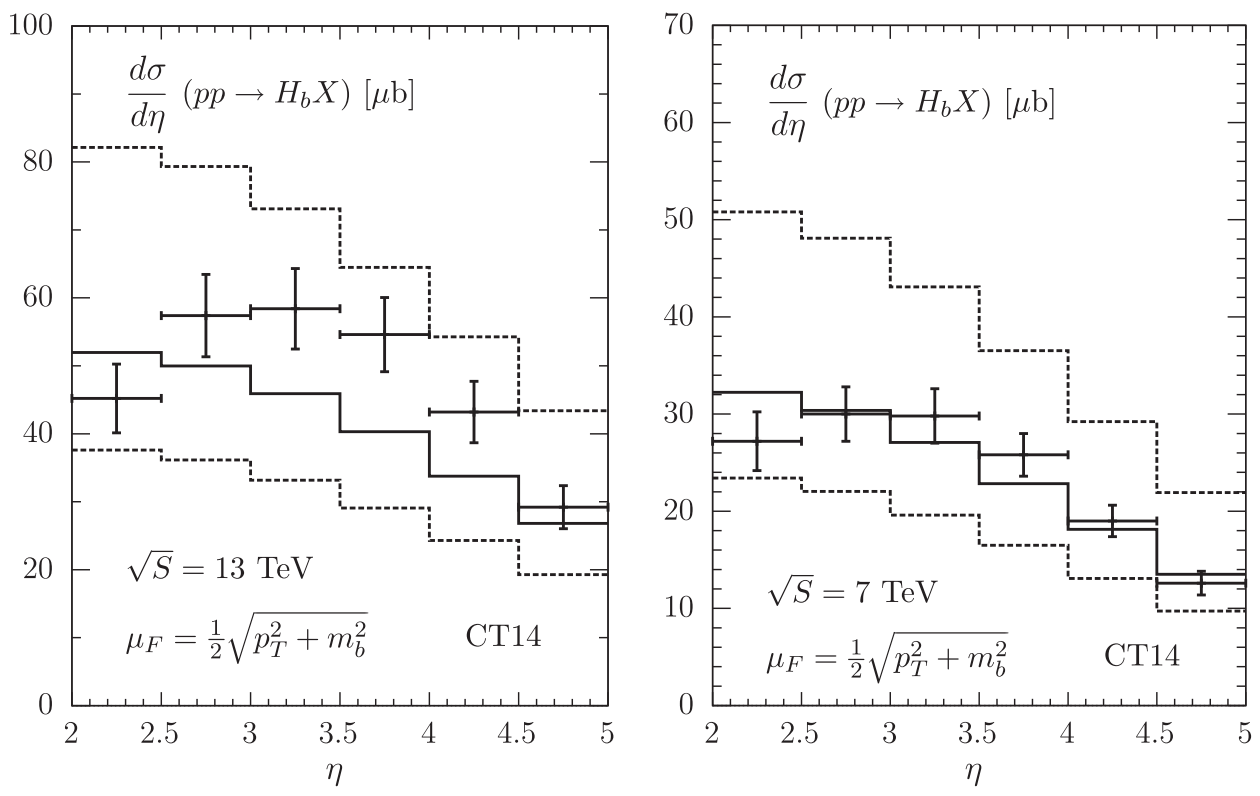

FIG. 2. Pseudorapidity distribution for $b$-hadron production compared with LHCb data [22], using CT14, $\sqrt{S}=13 \mathrm{TeV}$ (left) and $7 \mathrm{TeV}$ (right). The dashed-line histograms describe the theory uncertainty due to variations of the renormalization scale. The upper limit is found for $\mu_{R}=0.5 \sqrt{p_{T}^{2}+m_{b}^{2}}$, while the lower limit is for $\mu_{R}=2.0 \sqrt{p_{T}^{2}+m_{b}^{2}}$.

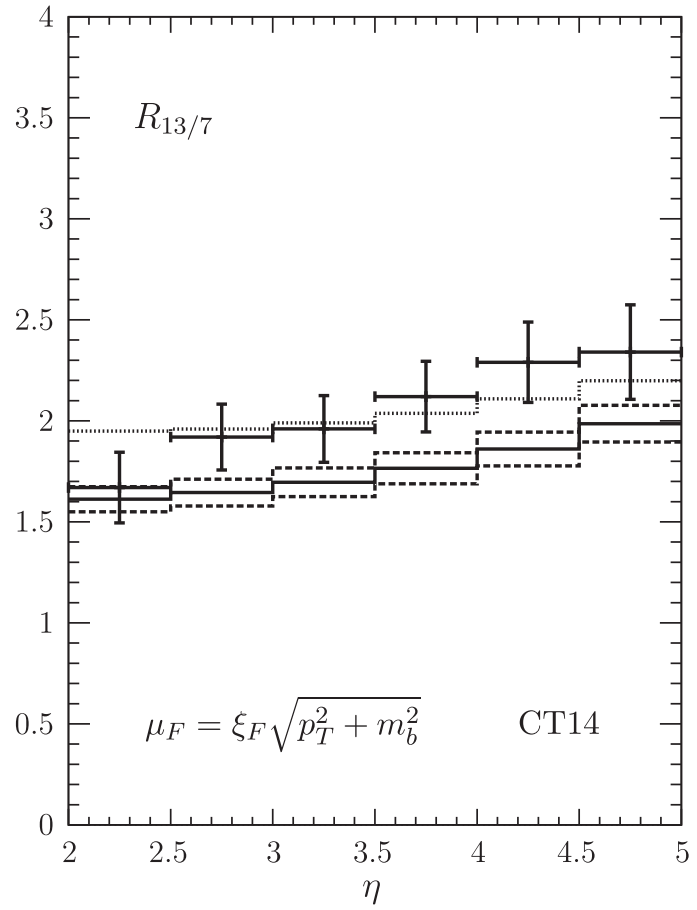

FIG. 3. The ratio of the pseudorapidity distributions for $\sqrt{S}=$ 13 and $7 \mathrm{TeV}$ for CT14 PDFs compared with LHCb data [22]. The full- and dashed-line histograms are calculated with $\xi_{F}=0.5$, as in Fig. 2. Its error band (dashed lines) shows the statistical uncertainty of the Monte Carlo integration. The dottedline histogram is obtained with $\xi_{F}=0.7$ for the cross section at $\sqrt{S}=13$ and with $\xi_{F}=0.5$ at $\sqrt{S}=7 \mathrm{TeV}$. is almost completely canceled in the ratio. In fact, our numerical calculation is dominated by the statistical uncertainty of the Monte Carlo integration: the error band shown in Fig. 3 (dashed-line histograms) represents this numerical uncertainty.

The cross section ratio $R_{13 / 7}$ is not affected by the large uncertainties from scale variations since one should use the same prescription for fixing the scales in the numerator and in the denominator. The theory uncertainties of the cross sections at the different center-of-mass energies cannot be treated like experimental errors, which have to be added in quadrature. We note that the agreement between data and theory could be improved if we used different scale choices for the different energies. As an example, we show in Fig. 3 how the ratio $R_{13 / 7}$ is changed if the cross section for $\sqrt{S}=13 \mathrm{TeV}$ is calculated with $\mu_{F}=0.7 \sqrt{p_{T}^{2}+m_{b}^{2}}$ instead of $\mu_{F}=0.5 \sqrt{p_{T}^{2}+m_{b}^{2}}$; the latter choice of scale is kept for the calculation at $\sqrt{S}=7 \mathrm{TeV}$. This prescription moves the ratio up in such a way that the data are perfectly well described in the upper five bins. Only the first bin shows a slight discrepancy. Of course, such a prescription to fix the factorization scale would be rather $a d h o c$ and we have no theoretical justification for it; however, it is also not forbidden by theory (see e.g., Ref. [35]). We do not attempt to introduce an additional $\eta$ dependence of scales, because such an approach would be even less well motivated.

It is well known that the theory uncertainty for the cross sections $d \sigma / d \eta$ is dominated by far by scale variations. These errors cancel, however, in the ratio $R_{13 / 7}$. 

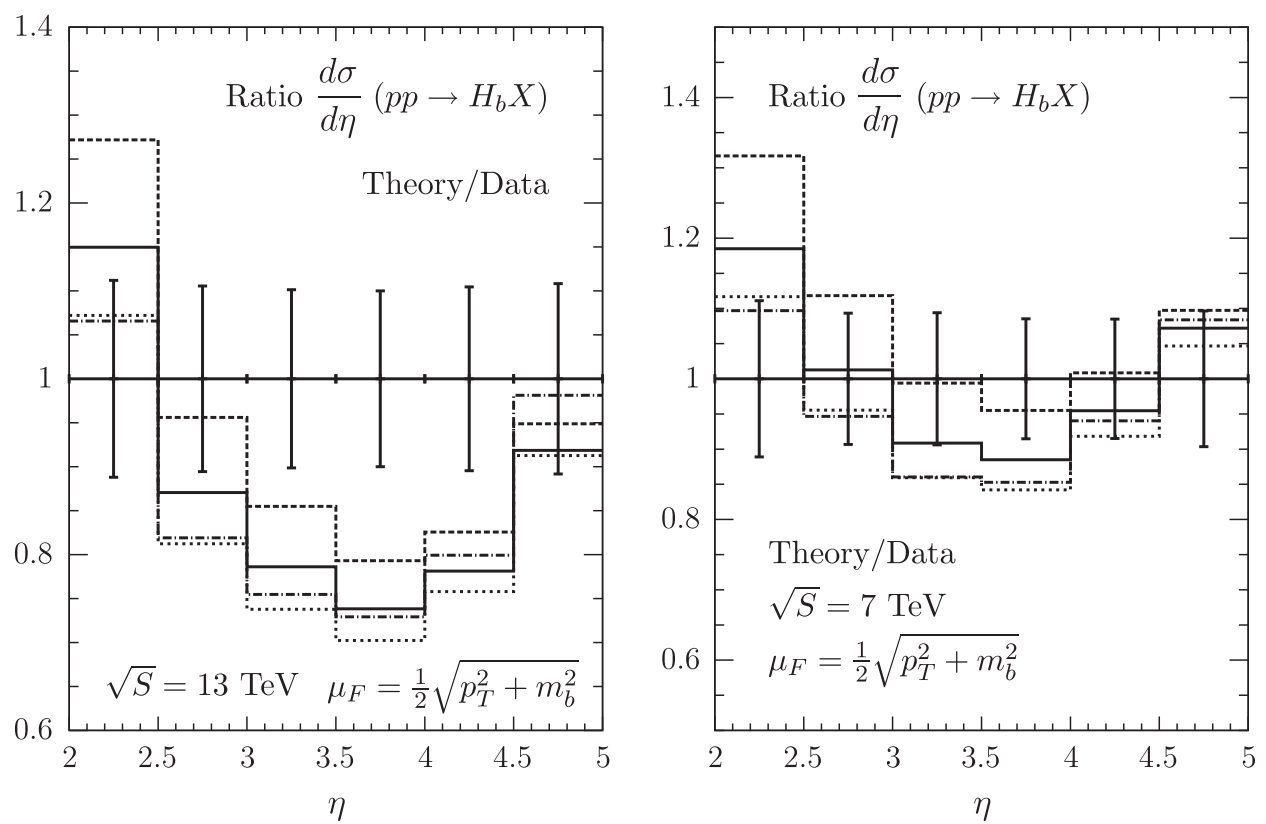

FIG. 4. PDF uncertainties of the pseudorapidity distributions for $b$-hadron production. The plot shows theory predictions normalized to the LHCb data [22] for $\sqrt{S}=13 \mathrm{TeV}$ (left) and $7 \mathrm{TeV}$ (right). Full line: CT14; dashed line: HERAPDF2.0; dotted line: NNPDF3.0; dash-dotted line: MMHT. For better visibility, scale uncertainties for the theory predictions are not shown. The corresponding uncertainty band would lie partly outside the range shown in the figure.

We therefore shall have a closer look at uncertainties due to the PDF input. First, in Fig. 4 we show results where we have also used the PDF parametrizations HERAPDF2.0 [25], MMHT [26] and NNPDF3.0 [27]. Here, the theory results for $d \sigma / d \eta$ are all normalized to the data and for better visibility we do not include the uncertainties due to scale variations. The corresponding error band would partly lie outside the range of values shown in this figure and the data points are all found inside the theory error band (see Fig. 2). We can see in Fig. 4 that the calculated cross sections do not depend strongly on the PDF input. This is, of course, not surprising since all PDF sets are based on fits to (almost) the same data.

Figure 5 shows the cross section ratio $R_{13 / 7}$ for the four different PDF sets. In the first few $\eta$ bins, the predictions are remarkably stable with respect to PDF variations. Only in the bin for the largest $\eta$ value can one observe that the PDF set MMHT leads to a roughly 5\% increase of $R_{13 / 7}$ which brings the prediction closer to the measured value, but this shift is smaller than the experimental uncertainty.

Assuming only ad hoc changes of the proton PDF cannot be expected to take full account of PDF uncertainties propagated from the data input on which the PDF fits are based. We therefore add results obtained by using uncertainty estimates which are now often also provided in a parametrized form by the PDF fitter collaborations. To be specific, we use the CT14 PDF set members with 28 pairs of parameter eigenvalue variations. One pair is particularly interesting since it describes an enhanced or suppressed

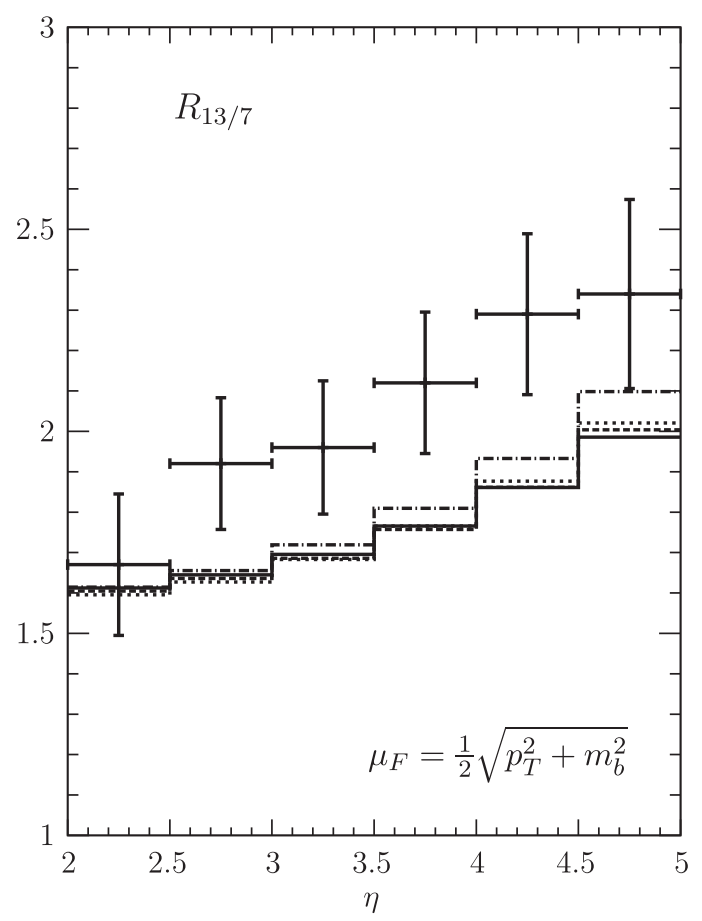

FIG. 5. The ratio of the pseudorapidity distributions for $\sqrt{S}=$ 13 and $7 \mathrm{TeV}$ as in Fig. 3 for different PDF sets. Full lines: CT14; dashed line: HERAPDF2.0; dotted line: NNPDF3.0; dash-dotted line: MMHT. The full line agrees with the one in Fig. 3, but note the magnified scale in this figure. 


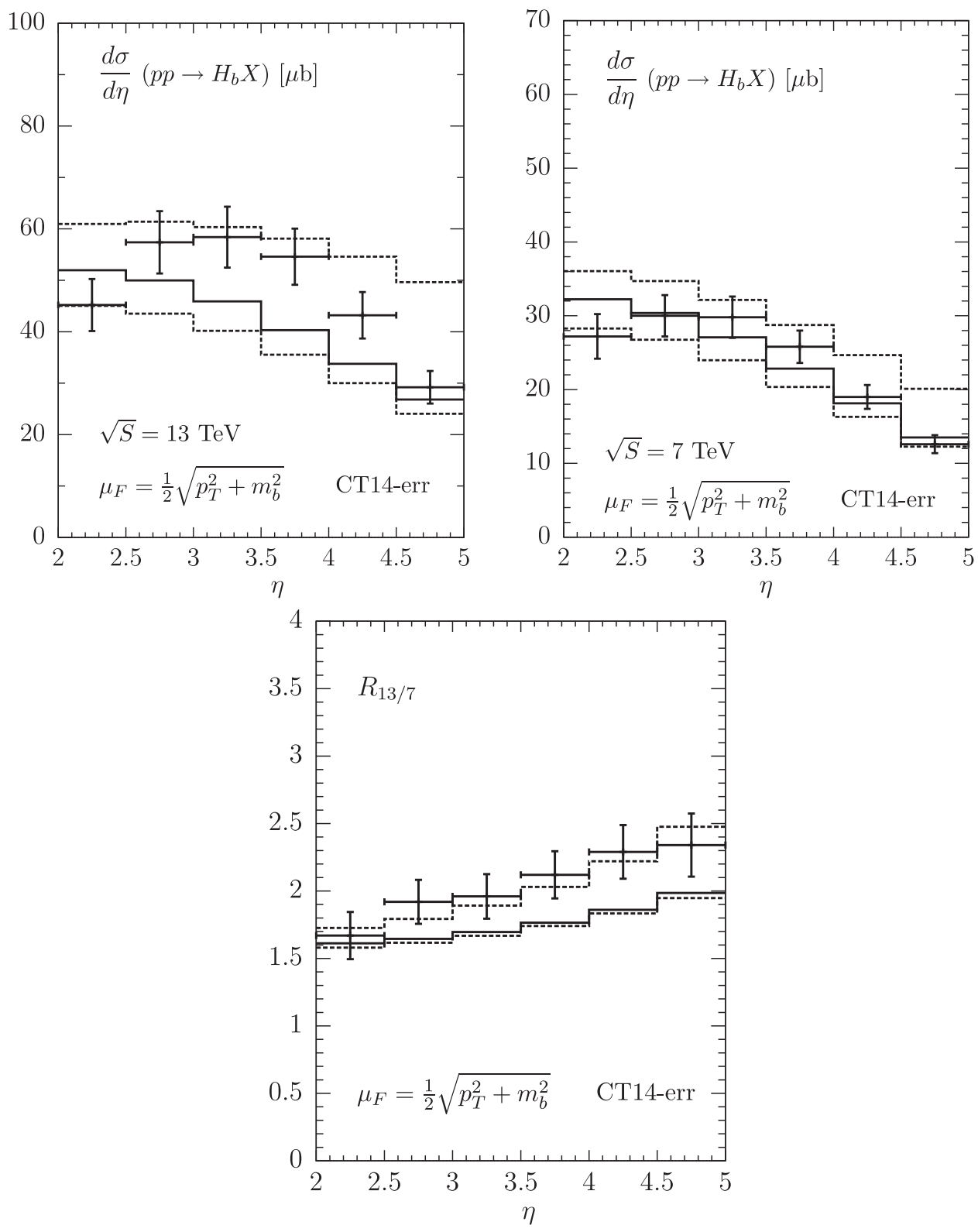

FIG. 6. Envelope of the PDF uncertainty for the pseudorapidity distributions using all 28 member pairs of CT14 compared with LHCb data [22]. Upper left: $d \sigma / d \eta$ at $\sqrt{S}=13 \mathrm{TeV}$; upper right: $d \sigma / d \eta$ at $\sqrt{S}=7 \mathrm{TeV}$; lower: the ratio $R_{13 / 7}$.

gluon distribution at very low $x$. Our results are shown in Fig. 6 for $d \sigma / d \eta$ at $\sqrt{S}=13 \mathrm{TeV}$ (upper left panel), $\sqrt{S}=7 \mathrm{TeV}$ (upper right panel), and for the ratio $R_{13 / 7}$ (lower panel). The dashed-line histograms represent the 90\% confidence level ${ }^{1}$ maximal and minimal variations obtained from the CT14 eigenvalue variations, evaluated following the prescription described in Ref. [28]. We find that indeed the PDF member pair 53 and 54 with an extreme choice of the gluon PDF has the strongest impact; the upward variation is dominated by one single eigenvector

\footnotetext{
${ }^{1}$ To obtain a $68 \%$ C.L. error band, one has to rescale the results by the factor $1 / 1.645$.
}

(member 53). We do not observe a significant difference in the shape of the differential cross sections $d \sigma / d \eta$ for these two CT14 member sets. The corresponding choice of the gluon distribution at low $x$ is possible since data still do not provide sufficient information. A similar observation has been made in a study of prompt neutrino fluxes from atmospheric charm production [36]. $d \sigma / d \eta$ depends strongly on the gluon PDF since $b$-hadron production is dominated by the subprocess $g g \rightarrow b \bar{b}$ at small $x \simeq 10^{-4}$ and at small scales. This agrees with the results of Ref. [37]. As can be seen in Fig. 6, the cross sections for $\sqrt{S}=13$ and $7 \mathrm{TeV}$ lie well inside the uncertainty band, while the ratio $R_{13 / 7}$ favors the upper limit of the CT14 error band. From 
our comparison we conclude that future PDF fits, in particular concerning the gluon PDF, could profit strongly from data for the $b$-quark production cross section ratio.

We have based this analysis on the CT14 PDF set, but the conclusion may be the same for other PDF sets. Following the PDF4LHC recommendations for LHC [38], one can also use a less conservative error estimate, not based on the envelope of one or more individual PDF error sets, but rather on a statistical combination of different PDF sets. Such an approach could be justified if one is interested in extracting information about the FFs where the PDF input should be kept fixed within uncertainties. We have checked that results obtained using the set PDF4LHC15_30 [38,39]

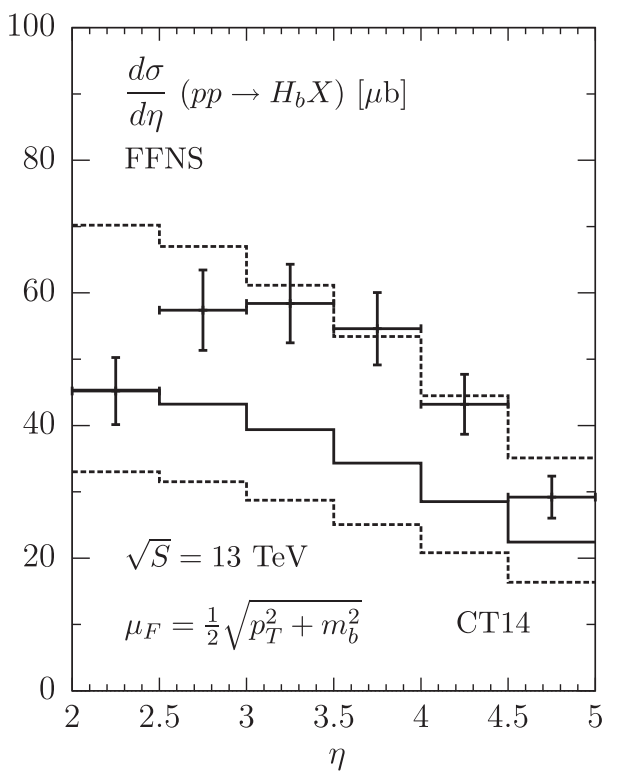

are consistent with our findings described above. The central values for the cross sections $d \sigma / d \eta$ are very similar to those shown above. At low rapidities the uncertainties are also close to the results from CT14; however, at large rapidities one finds uncertainties which are reduced by a factor of 2 to 3. The Hessian set PDF4LHC15_30 is based on 30 eigenvectors with, by definition, symmetric errors. Therefore the uncertainty for the ratio $R_{13 / 7}$ is negligible in this approach.

We finish this section with a comparison of the data with predictions obtained in the FFNS. In this approach, heavy quarks are produced at leading order only in the $g g$ channel and potentially large logarithms proportional to

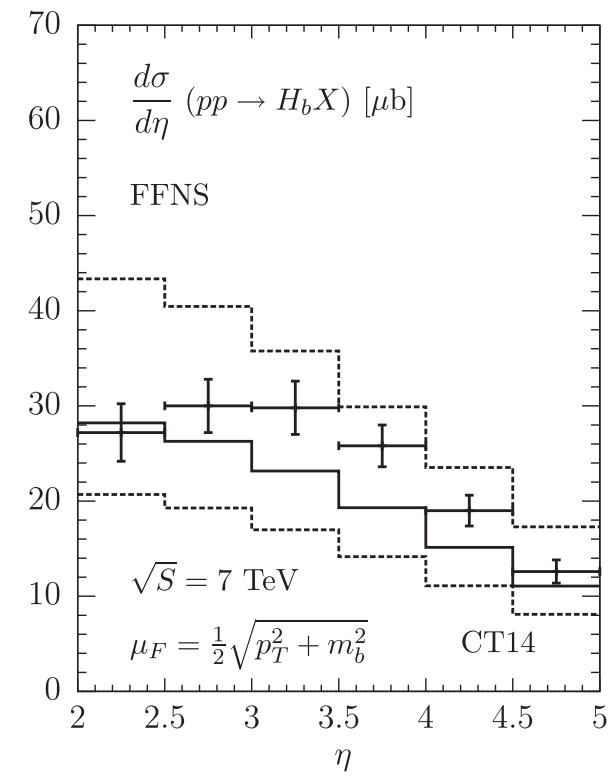

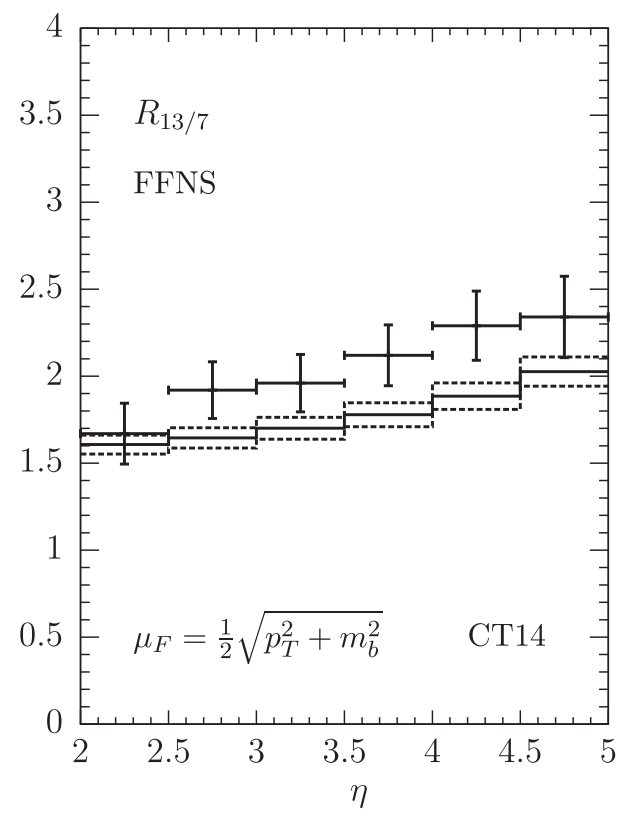

FIG. 7. Pseudorapidity distribution for $b$-hadron production compared with LHCb data [22] as in Fig. 2, but for the FFNS. Upper left: $d \sigma / d \eta$ at $\sqrt{S}=13 \mathrm{TeV}$; upper right: $d \sigma / d \eta$ at $\sqrt{S}=7 \mathrm{TeV}$; lower: the ratio $R_{13 / 7}$. We have used CT14 PDFs and no FFs. 
$\log \left(p_{T} / m_{b}\right)$ are not factorized into the PDFs and FFs. Consequently, there is no scale-dependent FF for the transition from $b$ quarks to $b$ hadrons. Since the LHCb data include all $b$ hadrons, we simply assume that the final state is given by a $b$ quark, i.e., we use a $\delta$ function as FF for the transition of a $b$ quark to a $b$ hadron. We use the CT14 PDFs including gluons and the light quarks $u, d, s$ and $c$ in the initial state, but no contribution from incoming $b$ quarks. The charm quark is treated as massless in this approach as before. In principle one should use a PDF set which is determined in the same framework of the FFNS with $n_{f}=4$, also including effects due to the nonzero charm mass [40], but from previous experience we do not expect large differences for such a more consistent approach. Results are shown in Fig. 7. The general picture looks quite similar to the GM-VFNS. The FFNS predictions are reduced by 15 to 20 percent, but the data are still within the theory uncertainty band. A comparison of the measured ratio $R_{13 / 7}$ with theory predictions in the FONLL framework [21] and using the NNPDF3.0 PDFs has also been presented in Ref. [22]. The results look very similar to ours as shown in Figs. 2 and 3. Large differences between the two schemes should indeed not be expected as discussed in Ref. [35].

\section{COMPARISON WITH CMS DATA}

There are also data from the CMS Collaboration for the ratio $R_{13 / 7}$ of cross sections for $b$-hadron production, albeit obtained from a measurement which includes only $B^{+}$meson final states, $p p \rightarrow B^{+} X$. Cross section data are available for $\sqrt{S}=13 \mathrm{TeV}$ [10] and $\sqrt{S}=7 \mathrm{TeV}$ [6]. $B^{+}$ mesons were identified using the decay $B^{+} \rightarrow J / \psi K^{+}$. The main difference with the LHCb analysis for $R_{13 / 7}$ is the kinematic range covered. The CMS Collaboration used the rapidity $y$ of the reconstructed $B^{+}$meson instead of the pseudorapidity as in the $\mathrm{LHCb}$ analysis. The ratio $R_{13 / 7}$ was obtained in the range $|y|<1.45$ and $|y|<2.1$, depending on different lower limits of the $p_{T}$ range: $p_{T}>$ $10 \mathrm{GeV}$ for $|y|<1.45$ and $p_{T}>17 \mathrm{GeV}$ for $|y|<2.1$, respectively [10].

We have calculated the cross section $d \sigma / d p_{T}$ at $\sqrt{S}=$ $13 \mathrm{TeV}$ for the nine $p_{T}$ bins in the region $10<p_{T}<$ $100 \mathrm{GeV}$ as in the data analysis: two $p_{T}$ bins with $p_{T} \in$ $[10,13] \mathrm{GeV}$ and $p_{T} \in[13,17] \mathrm{GeV}$ for $|y|<1.45$ and seven $p_{T}$ bins for $p_{T}>17 \mathrm{GeV}$ for $|y|<2.1$. For consistency with the discussion in the previous section, here we also use the factorization scale $\mu_{F}=0.5 \sqrt{p_{T}^{2}+m_{b}^{2}}$ and the renormalization scale $\mu_{R}=\sqrt{p_{T}^{2}+m_{b}^{2}}$ and vary $\mu_{R}$ up and down by a factor of 2 . The result is shown in the left panel of Fig. 8. The agreement between our calculation and the measured cross section is satisfactory. The measured values for $d \sigma / d p_{T}$ lie mostly close to the results for the scale choice which leads to the maximal cross section, i.e., $\mu_{R}=0.5 \sqrt{p_{T}^{2}+m_{b}^{2}}$. This relation between data and theory is similar to the comparison with FONLL predictions shown in Ref. [10]. Only the data point for the smallest $p_{T}$ bin lies well above the prediction and outside the theory error estimate.

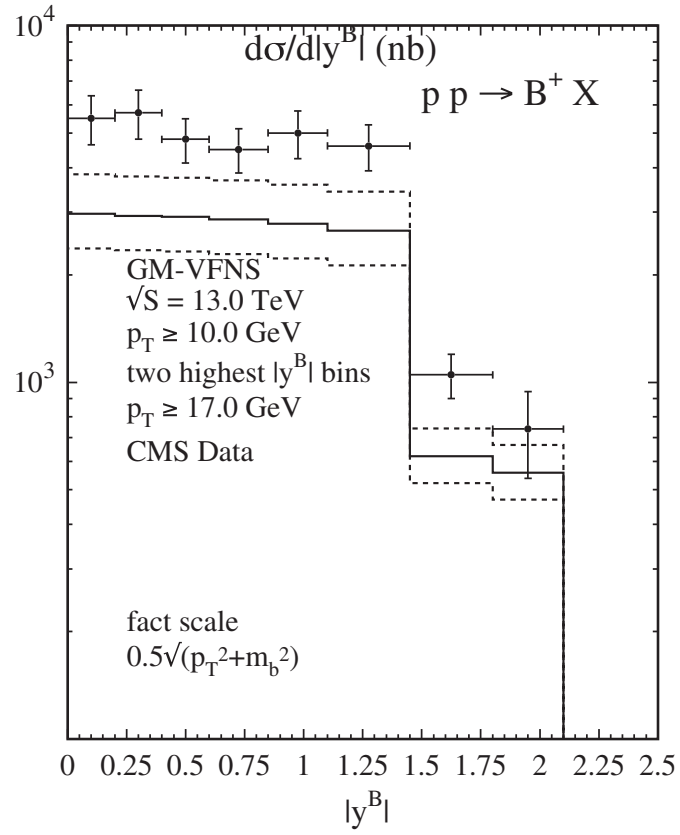

FIG. 8. $d \sigma / d p_{T}$ (left) and $d \sigma / d|y|$ (right) at $\sqrt{S}=13 \mathrm{TeV}$ for $B^{+}$production and comparison with data from the CMS Collaboration [10]. We have used $\mu_{F}=0.5 \sqrt{p_{T}^{2}+m_{b}^{2}}$ and $\mu_{R}=\sqrt{p_{T}^{2}+m_{b}^{2}}$. 

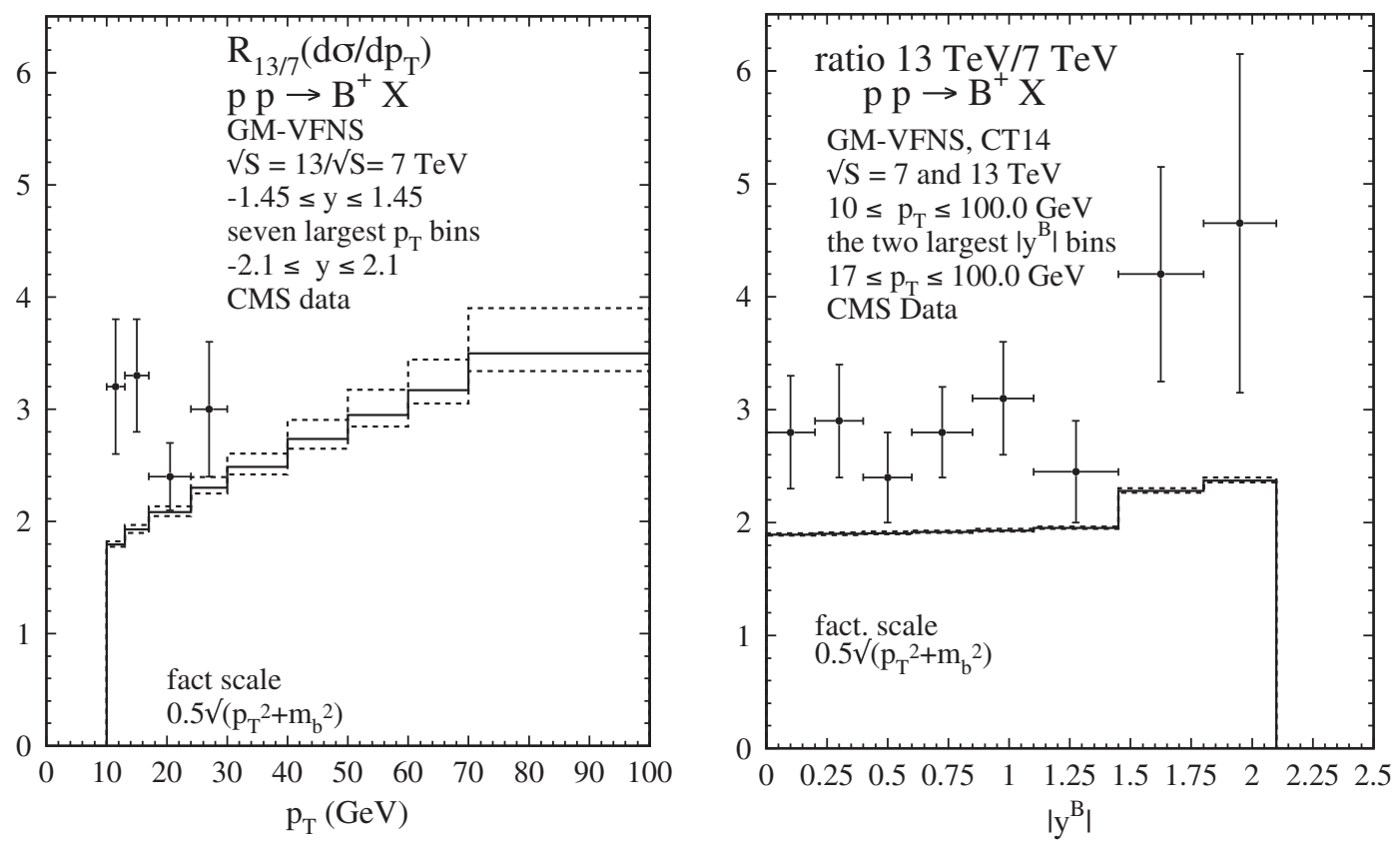

FIG. 9. The ratio $R_{13 / 7}$ of cross sections for $B^{+}$production and comparison with CMS data. The left panel is for the ratios of $d \sigma / d p_{T}$, while the right panel is for ratios of $d \sigma / d|y|$. The four data points in the left panel have been calculated from data given in Refs. [5,10]. We have used $\mu_{F}=0.5 \sqrt{p_{T}^{2}+m_{b}^{2}}$ and $\mu_{R}=\sqrt{p_{T}^{2}+m_{b}^{2}}$.

In the right panel of Fig. 8 we show the cross section $d \sigma / d|y|$ for eight $|y|$ bins integrated over the respective $p_{T}$ regions: $10<p_{T}<100 \mathrm{GeV}$ (first six lowest $|y|$ bins) and $17<p_{T}<100 \mathrm{GeV}$ (two highest $|y|$ bins). The data are compared to our predictions. The shape as a function of $|y|$ is very well reproduced, but the normalization of the cross section data is $60 \%$ higher than the calculated values. This is due to the fact that we have used the modified factorization scale $\mu_{F}=0.5 \sqrt{p_{T}^{2}+m_{b}^{2}}$. With the original choice $\mu_{F}=\sqrt{p_{T}^{2}+m_{b}^{2}}$, which is more appropriate for the cross section at larger $p_{T}$, we have found perfect agreement between data and theoretical predictions.

The ratio $R_{13 / 7}$ as a function of $p_{T}$ and $|y|$ were also reported in Ref. [10]. The data and the results of our
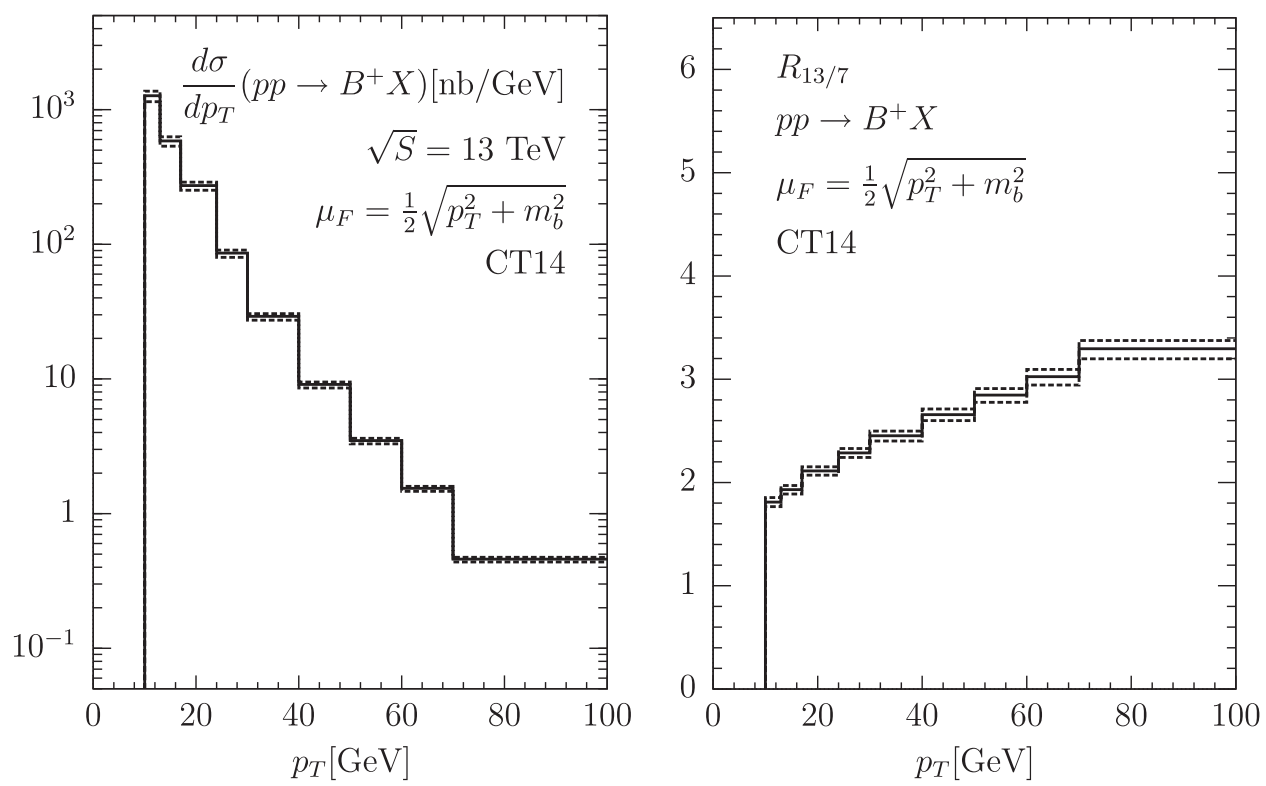

FIG. 10. PDF uncertainties obtained from the 52 members of the CT14 PDF parametrization for $d \sigma / d p_{T}$ at $\sqrt{S}=13 \mathrm{TeV}$ (left) and the ratio $R_{13 / 7}$ (right) for $B^{+}$production at CMS. 
calculation are shown in Fig. 9 (left frame for the $p_{T}$ dependence, right frame for the $|y|$ dependence). The four points for $p_{T}$ between 10 and $30 \mathrm{GeV}$ are larger than our predictions by approximately a factor of 1.6. Due to the rather large experimental errors, however, the disagreement in the CMS data is not very significant. The ratio $R_{13 / 7}$ as a function of $|y|$ is shown in Fig. 9 (right frame). The measured ratio for eight $|y|$ bins, also taken from Ref. [10], is again larger than the calculated ratio. Again, due to the large experimental uncertainties it is difficult to draw a definite conclusion from this comparison, similar to the case for the $p_{T}$ dependence of this ratio.

In the large- $p_{T}$ region covered by the CMS measurements we do not expect any significant dependence of the theoretical cross sections on different choices of input for the proton PDFs. Here we decided to test PDF uncertainties using the 26 pairs of eigenvalue variations of the CT14NLO PDFs [23]. We present the results in Fig. 10 for the cross section $d \sigma / d p_{T}$ for $p p \rightarrow B^{+} X$ (left part) and for the ratio $R_{13 / 7}$ (right part). We find rather small uncertainties from

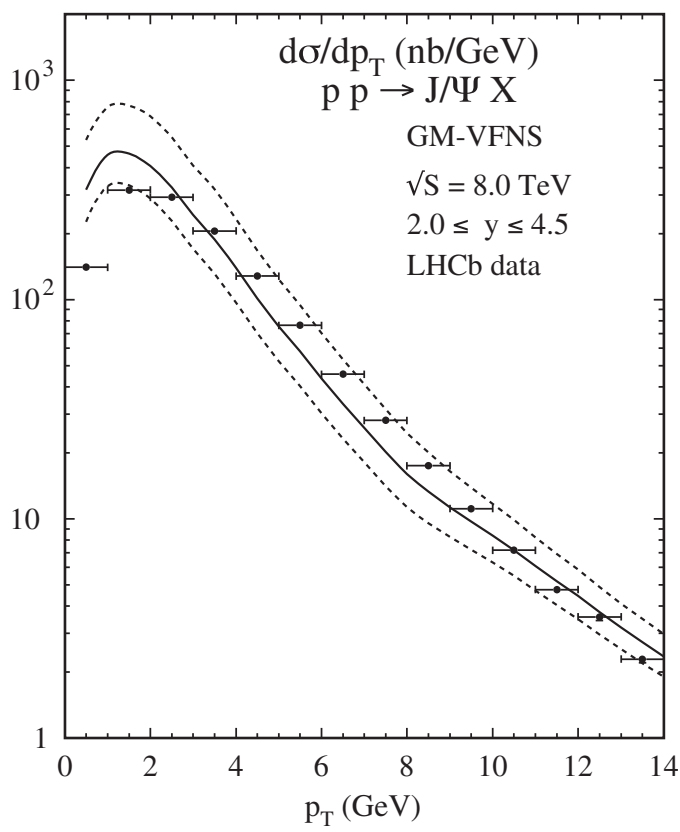

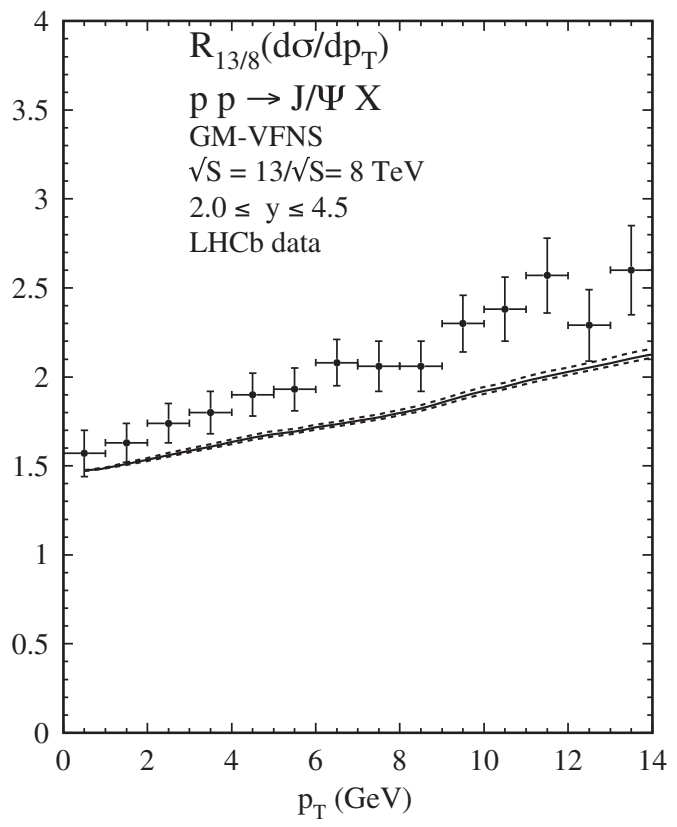

FIG. 11. The cross section $d \sigma / d p_{T}$ for inclusive $J / \Psi$ production from $b$ mesons compared with LHCb data [9] using CT14 at $\sqrt{S}=13 \mathrm{TeV}$ (upper left), $\sqrt{S}=8 \mathrm{TeV}$ (upper right), and their ratio (lower panel). 
this calculation, definitely much smaller than the uncertainties from scale variations for the theory prediction or from experimental sources for the data points.

\section{COMPARISON WITH LHCb DATA FOR INCLUSIVE $J / \Psi$ PRODUCTION FROM $b$ MESONS}

Another possibility to compare theoretical predictions with data for $b$-quark production with other final states is provided by a measurement by the LHCb Collaboration of inclusive $J / \Psi$ production from $b$-meson decays. Data are available for the ratio $R_{13 / 8}$ as a function of $p_{T}$ and rapidity $y$ at $\sqrt{S}=8 \mathrm{TeV}$ [41] and $\sqrt{S}=13 \mathrm{TeV}$ [9]. Some years ago, one of us together with Bolzoni and Kniehl considered this particular decay channel in the framework of the GM-VFNS incorporating theoretical input about the inclusive decay of $b$ hadrons into $J / \Psi$ mesons [42]. Predictions were compared with experimental data for $d \sigma / d p_{T}$ from the CDF, ALICE, ATLAS, CMS and LHCb collaborations existing at that time. In Ref. [42] it was found that the data collected by these collaborations agreed reasonably well with the theoretical predictions.
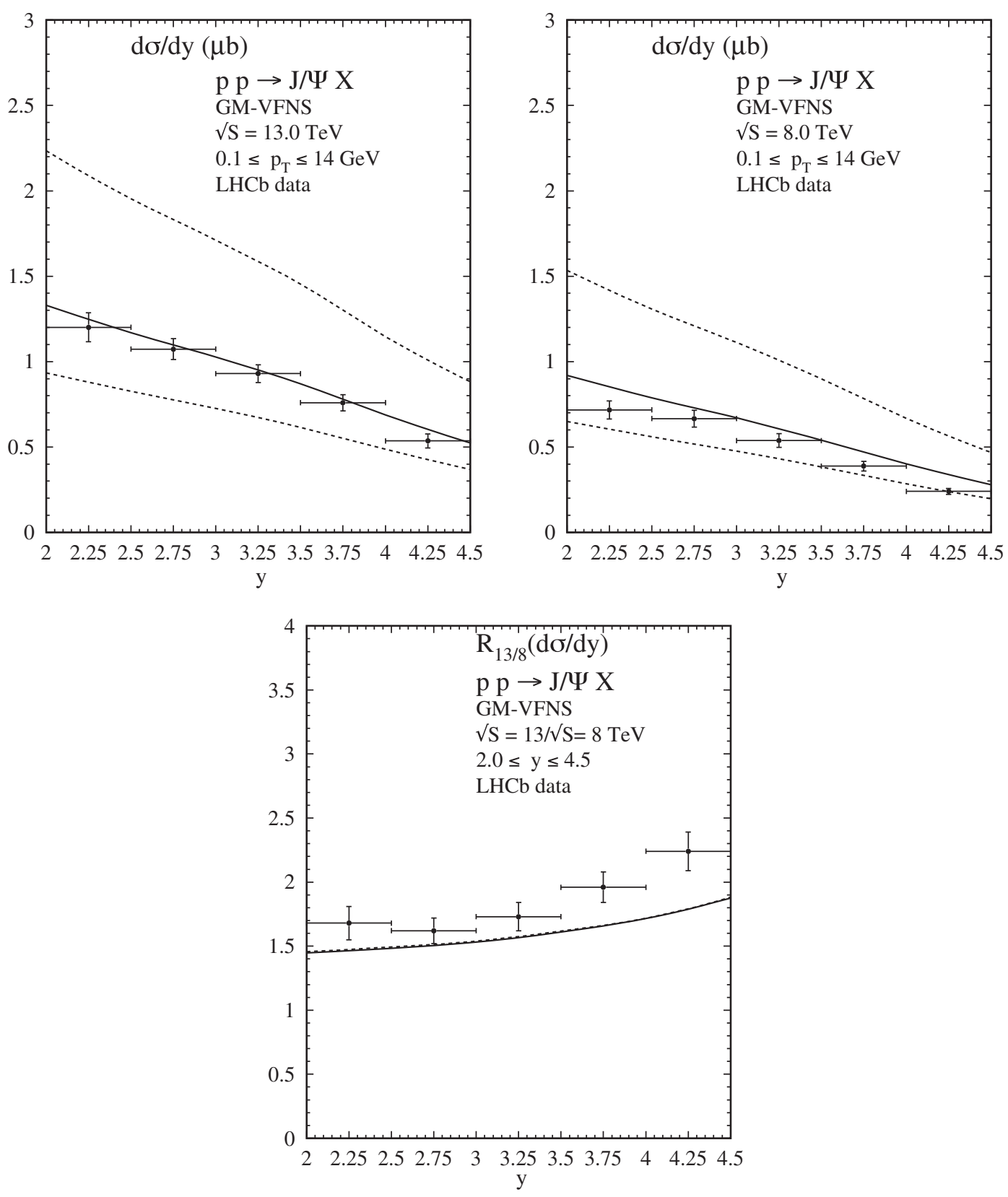

FIG. 12. The cross section $d \sigma / d y$ for inclusive $J / \Psi$ production from $b$ mesons compared with LHCb data [9] using CT14 at $\sqrt{S}=13 \mathrm{TeV}$ (upper left), $\sqrt{S}=8 \mathrm{TeV}$ (upper right), and their ratio (lower panel). 
Based on that earlier work we now calculate the transverse momentum and rapidity-dependent cross sections $d \sigma / d p_{T}$ and $d \sigma / d y$ for $\sqrt{S}=8 \mathrm{TeV}$ and $\sqrt{S}=13 \mathrm{TeV}$ and study the ratio $R_{13 / 8}$ as a function of $p_{T}$ and $y$ and compare with the experimental data presented in Ref. [9]. In contrast to the earlier work on $J / \Psi$ production from $b$-hadron decay we now use the modified scale as described in Sec. II. We expect that the scale choice $\mu_{F}=0.5 \sqrt{p_{T}^{2}+m_{b}^{2}}$ is also required to achieve a reasonable description of data at low $p_{T}$. Our results for $d \sigma / d p_{T}$ as a function of $p_{T}$ for $\sqrt{S}=13 \mathrm{TeV}$ and $\sqrt{S}=8 \mathrm{TeV}$ are shown in Fig. 11 in the left and right upper panels, respectively. The theory results are compared with data taken from Ref. [9] (for $\sqrt{S}=13 \mathrm{TeV}$ ) and Ref. [41] (for $\sqrt{S}=8 \mathrm{TeV}$ ). The experimental data points lie well inside the theoretical range which is determined from scale variations as usual. On average, the range between the default scale and the scale choice $\mu_{R}=\mu_{F}$ which leads to the maximal cross section is preferred by the data. The ratio $R_{13 / 8}$ for $d \sigma / d p_{T}$ as a function of $p_{T}$ is shown in the lower panel of Fig. 11. The theoretical prediction for $R_{13 / 8}$ increases from 1.5 to about 2.2 and lies systematically below all data points, even when taking into account experimental uncertainties. The theoretical prediction for the ratio $R_{13 / 8}$ has essentially no scale dependence since we take the ratio of cross sections with identical scale parameters in the numerator and denominator. Numerical uncertainties are small and not shown in the figure.

The rapidity dependence of the cross section, $d \sigma / d y$, in the range $2.0 \leq y \leq 4.5$ integrated over the transverse momentum in the range $0 \leq p_{T} \leq 14 \mathrm{GeV}$ is presented in Fig. 12. The upper left and right panels show the cross section as a function of $y$ for $\sqrt{S}=13$ and $8 \mathrm{TeV}$, respectively. Corresponding experimental data were given in Refs. $[9,41]$ in five bins of size $\Delta y=0.5$. While the $13 \mathrm{TeV}$ data agree perfectly well with predictions for the default scale, the data at $\sqrt{S}=8 \mathrm{TeV}$ lie somewhat below the prediction for the default scale, but still inside the theory uncertainty band whose lower limit is obtained with the choice $\mu_{R}=4 \mu_{F}$. The theoretical result for the ratio $R_{13 / 8}$ as a function of $y$ is shown in the lower panel of Fig. 12. Here, the agreement between theory and data is rather marginal. A better agreement could be found if the prediction for $d \sigma / d y$ at $\sqrt{S}=8 \mathrm{TeV}$ was slightly lower. In the present case, the strongest deviation between data and theory is in the last bins at the high $y$ values.

\section{CONCLUSIONS}

As a conclusion from our analysis of predictions for inclusive $b$-hadron production in $p p$ collisions at the LHC we can state that the majority of experimental data for the cross sections, differential in transverse momentum or in (pseudo)rapidity, are reasonably well described by theory. This is mainly due to the large theory uncertainty due to variations of the renormalization scale. An exception is maybe seen in the comparison with $B^{+}$meson production data from the CMS Collaboration at $\sqrt{S}=13 \mathrm{TeV}$ where the cross section data are somewhat higher than theory.

In all the cases we found some tension between data and theory if the comparison is based on the cross section ratio for different center-of-mass energies. The data prefer slightly higher values of $R_{13 / 7}$ for $b$-hadron production measured by the LHCb Collaboration and for $B^{+}$-meson production measured by the CMS Collaboration. Also the $\mathrm{LHCb}$ data for the ratio $R_{13 / 8}$ of $J / \Psi$ production through decays from $b$ mesons is higher than our predictions.

We found that the cross section ratios are remarkably stable with respect to variations of the renormalization scale if one follows the widespread assumption that the renormalization and factorization scales should depend only on the transverse momentum of the observed hadron. Our calculations show that a weakly $\sqrt{S}$ dependent choice of scales would further reduce the significance of the slight disagreement. Such a scale choice is theoretically neither well motivated, nor completely unreasonable.

As a consequence of the stability of theoretical predictions with respect to scale variations, one can also conclude that the cross section ratios will serve as important input for improved determinations of PDF parametrizations. This was exemplified in particular in a comparison with members of the CT14 PDF parametrizations. Future fits could result in an improved knowledge of the gluon PDF at low $x$ and, correlated with the low $p_{T}$ of the data, at low scale. In order to test such a possibility further it will be important to also include data at larger $p_{T}$. It would also be helpful if the LHCb Collaboration could provide their data for $b$-hadron production with a higher value of the minimum transverse momentum. An extension of the kinematic range, both in transverse momentum and in (pseudo)rapidity, is also important to test whether data from different experiments and with different $b$-hadron final states are compatible with each other. 
[1] D. Acosta et al. (CDF Collaboration), Phys. Rev. D 71, 032001 (2005).

[2] A. Abulencia et al. (CDF Collaboration), Phys. Rev. D 75, 012010 (2007).

[3] A. M. Sirunyan et al. (CMS Collaboration), Phys. Rev. Lett. 119, 152301 (2017).

[4] G. Aad et al. (ATLAS Collaboration), J. High Energy Phys. 10 (2013) 042.

[5] V. Khachatryan et al. (CMS Collaboration), Phys. Rev. Lett. 106, 112001 (2011).

[6] S. Chatrchyan et al. (CMS Collaboration), Phys. Rev. Lett. 106, 252001 (2011).

[7] S. Chatrchyan et al. (CMS Collaboration), Phys. Rev. D 84, 052008 (2011).

[8] R. Aaij et al. (LHCb Collaboration), J. High Energy Phys. 04 (2012) 093.

[9] R. Aaij et al. (LHCb Collaboration), J. High Energy Phys. 10 (2015) 172; 05 (2017) 063(E).

[10] V. Khachatryan et al. (CMS Collaboration), Phys. Lett. B 771, 435 (2017).

[11] S. Chatrchyan et al. (CMS Collaboration), Phys. Lett. B 714, 136 (2012).

[12] R. Aaij et al. (LHCb Collaboration), Chin. Phys. C 40, 011001 (2016).

[13] M. Cacciari, S. Frixione, N. Houdeau, M. L. Mangano, P. Nason, and G. Ridolfi, J. High Energy Phys. 10 (2012) 137.

[14] B. A. Kniehl, G. Kramer, I. Schienbein, and H. Spiesberger, Phys. Rev. D 84, 094026 (2011).

[15] B. A. Kniehl, G. Kramer, I. Schienbein, and H. Spiesberger, Eur. Phys. J. C 75, 140 (2015).

[16] B. A. Kniehl, G. Kramer, I. Schienbein, and H. Spiesberger, Phys. Rev. D 71, 014018 (2005).

[17] B. A. Kniehl, G. Kramer, I. Schienbein, and H. Spiesberger, Eur. Phys. J. C 41, 199 (2005).

[18] I. Helenius and H. Paukkunen, J. High Energy Phys. 05 (2018) 196.

[19] R. Aaij et al. (LHCb Collaboration), Phys. Rev. Lett. 118, 052002 (2017).

[20] CERN Courier 56 (2016), p. 12.

[21] M. Cacciari, M. L. Mangano, and P. Nason, Eur. Phys. J. C 75, 610 (2015).
[22] R. Aaij et al. (LHCb Collaboration), Phys. Rev. Lett. 118, 052002 (2017); 119, 169901(E) (2017).

[23] S. Dulat, T.-J. Hou, J. Gao, M. Guzzi, J. Huston, P. Nadolsky, J. Pumplin, C. Schmidt, D. Stump, and C.-P. Yuan, Phys. Rev. D 93, 033006 (2016).

[24] A. Buckley, J. Ferrando, S. Lloyd, K. Nordström, B. Page, M. Rüfenacht, M. Schönherr, and G. Watt, Eur. Phys. J. C 75, 132 (2015).

[25] H. Abramowicz et al. (H1 and ZEUS Collaborations), Eur. Phys. J. C 75, 580 (2015).

[26] L. A. Harland-Lang, A. D. Martin, P. Motylinski, and R. S. Thorne, Eur. Phys. J. C 75, 204 (2015).

[27] R. D. Ball et al. (NNPDF Collaboration), J. High Energy Phys. 04 (2015) 040.

[28] H. L. Lai, M. Guzzi, J. Huston, Z. Li, P. M. Nadolsky, J. Pumplin, and C.-P. Yuan, Phys. Rev. D 82, 074024 (2010).

[29] B. A. Kniehl, G. Kramer, I. Schienbein, and H. Spiesberger, Phys. Rev. D 77, 014011 (2008).

[30] A. Heister et al. (ALEPH Collaboration), Phys. Lett. B 512, 30 (2001).

[31] G. Abbiendi et al. (OPAL Collaboration), Eur. Phys. J. C 29, 463 (2003).

[32] K. Abe et al. (SLD Collaboration), Phys. Rev. Lett. 84, 4300 (2000).

[33] K. Abe et al. (SLD Collaboration), Phys. Rev. D 65, 092006 (2002); 66, 079905(E) (2002).

[34] R. Aaij et al. (LHCb Collaboration), J. High Energy Phys. 08 (2013) 117.

[35] F. Maltoni, G. Ridolfi, and M. Ubiali, J. High Energy Phys. 07 (2012) 022; 04 (2013) 095(E).

[36] M. Benzke, M. V. Garzelli, B. Kniehl, G. Kramer, S. Moch, and G. Sigl, J. High Energy Phys. 12 (2017) 021.

[37] R. Gauld, J. High Energy Phys. 05 (2017) 084.

[38] J. Butterworth et al., J. Phys. G 43, 023001 (2016).

[39] J. Gao and P. Nadolsky, J. High Energy Phys. 07 (2014) 035.

[40] J. Blümlein, A. De Freitas, C. Schneider, and K. Schönwald, Phys. Lett. B 782, 362 (2018).

[41] R. Aaij et al. (LHCb Collaboration), J. High Energy Phys. 06 (2013) 064.

[42] P. Bolzoni, B. A. Kniehl, and G. Kramer, Phys. Rev. D 88, 074035 (2013). 